ISSN: 0213-3563

https://doi.org/10.14201/azafea202123167187

\title{
EL PERFECCIONISMO POSTKANTIANO Y LA IZQUIERDA HEGELIANA
}

Post-Kantian Perfectionism and Hegelian Left

Douglas Moggach

Universidad de Ottawa (Canadá)

Recibido: 31 de mayo de 2021

Aceptado: 7 de agosto de 2021

\section{RESUMEN}

La ética política de la Escuela hegeliana se caracteriza por el perfeccionismo post-kantiano, un enfoque que difiere de las teorías perfeccionistas precedentes en que tiene por objeto la promoción de la libertad y las condiciones de su ejercicio. Las ideas de actividad espontánea o iniciada por sí misma desplazan los puntos de vista antiguos de la felicidad o la prosperidad (eudaimonia), basados en concepciones fijas de la naturaleza humana. Las nuevas teorías están también pendientes de los intereses conflictivos en la sociedad civil moderna emergente y de la necesidad de transformar sus prácticas e instituciones. Dentro de este enfoque se distinguen dos variantes en la década de 1840, las de Bauer y Marx, de las cuales aquí se examinan sus respectivos compromisos normativos y metaéticos.

Palabras clave: perfeccionismo; Leibniz; Kant; Escuela hegeliana; Bruno Bauer; Karl Marx.

\section{ABSTRACT}

The political ethics of the Hegelian School is characterized by post-Kantian perfectionism, an approach which differs from earlier perfectionist theories because it takes as its object the promotion of freedom and the conditions of its exercise. Ideas of spontaneous or self-initiated activity displace older views of happiness or thriving (eudaimonia), based on fixed conceptions of human nature. The new theories are also attentive to conflicting interests 
in emergent modern civil society, and the need to transform its institutions and practices. Two of the variants within this approach in the 1840's are distinguished, those of Bauer and Marx, and their respective metaethical and normative commitments are examined.

Key words: perfectionism; Leibniz; Kant; Hegelian School; Bruno Bauer; Karl Marx.

La categoría del perfeccionismo postkantiano se ha venido aplicando recientemente a programas éticos como los de Fichte o Schiller y a los de la izquierda hegeliana, los cuales, en la estela de las críticas de Kant, asumen como fin central la promoción de la libertad y sus condiciones materiales, políticas y sociales (Moggach, 2009; 2011). El carácter postkantiano de estos programas deriva del giro que se produce desde la eudaimonia, la felicidad o la satisfacción de necesidades (algo típico de los perfeccionismos más antiguos desde Aristóteles y también de su promulgación moderna, en la línea de Leibniz, por su intérprete Christian Wolff) (Wolff, 1969 [1754]) hacia la libertad y la autonomía como valores fundamentales. Las teorías perfeccionistas sostienen en general que el desarrollo de ciertas capacidades constituye un valor ético intrínseco y sobrevenido (Leopold, 2007: 185). Para el perfeccionismo postkantiano, el objetivo no es imponer alguna concepción sustantiva de la vida buena, como habían hecho perfeccionismos anteriores, sino asegurar y mejorar las condiciones para el ejercicio de la libertad y eliminar obstáculos a la autodeterminación. Así pues, la especificidad del enfoque postkantiano consiste en su búsqueda del modo de promover la capacidad para la actividad libre en sí misma, más que en cualquier visión determinada de los requisitos para lograr el carácter virtuoso o la vida feliz. En lugar de entrar en declive tras la famosa y potencialmente devastadora crítica de Kant al perfeccionismo en la ética y en el pensamiento político, el perfeccionismo asumió nuevas formas, incluso entre kantianos como Schiller y Fichte, así como, cabe añadir, en Hegel y sus seguidores en la década de 1840 y posteriormente.

Para anticipar aquí el argumento, tres cambios simbolizan el movimiento hacia este nuevo perfeccionismo. En primer lugar, mientras el perfeccionismo prekantiano seguía a Aristóteles en la promoción de la felicidad o del correcto desarrollo, el perfeccionismo después de Kant tomó la libertad como su meta, o bien el asegurarse las condiciones para la actividad libre en el mundo 
objetivo. La libertad requiere que cada individuo sea capaz de perseguir una concepción particular de felicidad sin ninguna imposición autoritaria. El carácter perfeccionista de este enfoque reside en su compromiso con la "creación social” (Maesschalck, 1996) para asegurar y aumentar la práctica de la libertad y eliminar obstáculos a esta: las acciones son validadas en función de su contribución a estos fines. En segundo lugar, como una definición ampliada de lo que es la actividad libre, se descubren las ideas de la formatividad y la auto-formación: mientras el perfeccionismo prekantiano estaba típicamente asentado en una consideración teleológica de la naturaleza y una finalidad esencial o consustancial a los entes, el perfeccionismo después de Kant miró más bien hacia la idea de los propósitos escogidos por uno mismo, la libre auto-constitución y la autodeterminación. La naturaleza humana no es algo fijo, sino históricamente construido. En tercer lugar, mientras el perfeccionismo prekantiano tendía a ver los intereses humanos como armoniosos y complementarios, el perfeccionismo después de Kant reconoció la diversidad y el pluralismo, y desde luego la fragmentación y disrupción de la vida moderna, e intentó incorporar esta conflictiva diversidad a su consideración de una posible moralidad y del progreso político. De esta forma, el perfeccionismo postkantiano hizo suya la poderosa crítica de Kant a la tradición existente y llevó al perfeccionismo a renovarse a la luz de ella.

Mi tesis es que las teorías políticas desarrolladas en la escuela hegeliana de la década de 1840 representan diversas manifestaciones de las ideas perfeccionistas postkantianas. Karl Marx también comparte este enfoque con sus contemporáneos de la izquierda hegeliana, aunque el giro de la felicidad a la libertad se da en diferente gradación entre ellos. Este giro está mucho más marcado en Bruno Bauer que en Marx, por ejemplo, quien combina enfoques kantianos y naturalistas del yo y su actividad; aun así, esto es definitorio en las primeras concepciones de Marx sobre la emancipación y el trabajo. Los conceptos idealistas del auto-movimiento, la acción espontánea o autooriginada, así como la distinción kantiana entre la razón pura práctica y la empírica, forman el trasfondo esencial de este pensamiento incluso cuando, como en el caso de Marx, se traduce a un léxico más materialista. Las diferentes maneras de comprender estos conceptos y sus implicaciones normativas subrayan las divergencias políticas entre el republicanismo alemán de inspiración hegeliana y el socialismo en 1848.

Para entender la importancia del concepto de libertad como espontaneidad en esta tradición, es necesario remontarse hasta su fuente en la filosofía de Leibniz, que, como argumentó elocuentemente Ernst Cassirer, es el 
verdadero origen del idealismo alemán, en el cual se inserta el perfeccionismo postkantiano (Cassirer, 1962 [1902]): 457-458). Leibniz hace de la relación entre la libertad y la justicia un nexo central en sus reflexiones sobre filosofía política, hasta el punto de permanecer como paradigma en el pensamiento alemán posterior. En su escrito de juventud, Nova Methodus Discendae Docendaeque Jurisprudentiae (1667), Leibniz deriva obligaciones jurídicas de los tres principios de la ley romana, reinterpretados a la luz de su filosofía en ciernes: neminem laedere, suum cuique tribuere, honeste (pie) vivere. Leibniz lee el primer principio como la obligación de ejercer la libertad de uno mismo absteniéndose de obstaculizar la libertad de los demás. En otros textos (Leibniz, 1991 [1720]: \$10-\$13; Rutherford, 2005), Leibniz definirá la espontaneidad como el constante cambio activo del sujeto (o mónada) al ejecutar sus propios imperativos interiores, gobernado por una ley inmanente de desarrollo específica de cada cual. Espontaneidad para Leibniz es la capacidad de los sujetos para ser la causa auto-iniciada del cambio en sí mismos y en el mundo fenoménico, así como para evaluar y validar estos cambios; tales sujetos autodeterminados no son meramente moldeados por causas externas, como en el materialismo de la Ilustración, sino que integran estas causas en sus acciones de una manera propia y creativa. Leibniz no rechaza el nuevo materialismo, pero lo consigna en el lugar que le corresponde en el reino de los fenómenos o fuerzas derivadas, del cual él distingue una esfera fundacional de la libertad; la actividad teleológica se lleva a cabo no como respuesta a causas mecanicistas, sino por la visión de la idea de un bien realizable. Esta conceptualización permite una reconstrucción del principio de la primera ley natural como el derecho a la espontaneidad, a la actividad libremente auto-orientada y al constante cambio auto-dirigido; y este principio ha sido interpretado en trabajos recientes como generador de un carácter más bien deontológico que consecuencialista en cuanto a su fundamento justificativo (Leibniz, 1990 [1667): \$73-\$75). El segundo principio de la ley natural para contribuir a la felicidad y la perfección del conjunto, es, según Leibniz, un principio de justicia distributiva; se admiten varios sistemas de organización de la propiedad y del orden social en distintas circunstancias empíricas, pero estos sistemas permisibles están todos sujetos a una doble restricción: por un lado, a la del primer principio, consistente en respetar el derecho a la espontaneidad de todos los miembros de la comunidad y, por otro lado, a la exigencia de perfección, entendida como la contribución de estos sistemas al bienestar y a la eudaimonia del conjunto. El tercer principio, para progresar en el amor y conocimiento de Dios, compromete a los individuos en el 
proceso de realización del mejor de los mundos posibles como una misión moral, lo cual implica un cambio y desarrollo continuo, animado por un conatus interior que se dirige a alcanzar niveles cada vez mayores de perfección, o a la manifestación de todas las propiedades que estarían implícitas en una totalidad armoniosa (Schneider, 1967).

Es importante observar más de cerca una de las ulteriores articulaciones de la posición de Leibniz, la más apropiada para captar el contraste fundamental entre los sistemas perfeccionistas anteriores y posteriores a Kant. Christian Wolff (1679-1754), el divulgador y sistematizador de Leibniz, desarrolla una ética perfeccionista como base para un Estado fuertemente intervencionista (Wolff, 1969 [1754]; Stipperger, 1984; Schneewind, 1998). El Estado debe actuar para asegurar la perfección porque los sujetos por sí mismos son incapaces de alcanzar el máximo nivel de desarrollo físico, intelectual y espiritual. Los sujetos precisan formas institucionalizadas en las que cooperar suministradas por el Estado. Para Wolff el primer principio leibniziano no se sostiene por sí solo. La espontaneidad subjetiva requiere una dirección exógena si quiere alcanzar su meta de perfección. Esta es la base de su llamada (destinada a Federico el Grande de Prusia y otros monarcas reformistas de su tiempo) a un despotismo ilustrado intervencionista (Tribe, 1988), dirigido a promover el óptimo desarrollo de todos en cada aspecto de la vida. Los derechos son considerados títulos a los que se puede y debe renunciar en aras de la conquista de perfección (Klippel, 1998: 81). Wolff reconfigura así los principios jurídicos fundamentales de Leibniz haciendo el primero condicional con respecto al segundo, a la red institucionalizada sólo a través de la cual la espontaneidad puede ser activada y dirigida hacia su objetivo: el tercer principio o idea de progreso. Aun así, el segundo principio mantiene su estatus normativo como guía para legitimar la acción pública. Justifica las políticas del despotismo ilustrado no mediante su contribución a la libertad de los sujetos, sino a su felicidad y perfección.

Se puede entender esta posición y su contenido concreto en su contexto histórico. Si los Estados centralistas de Inglaterra y Francia pudieron perseguir la expansión de sus economías a través de la práctica del mercantilismo, para el cual las colonias sirvieron como garantía de fuentes de materias primas y mercados para la producción, los débiles y divididos Estados alemanes del s. XVIII tenían que buscar otras salidas. El pensamiento de Wolff tiene afinidades muy próximas con una forma específica de economía política, conocida como cameralismo, que surgió en estas circunstancias en Europa central. El cameralismo es una alternativa al mercantilismo en condiciones 
en las que no había colonias y las fronteras entre Estados eran relativamente porosas para los movimientos de población. El objetivo es formar y retener a trabajadores y artesanos altamente cualificados, así como desarrollar recursos locales y vínculos comerciales: la expansión de las fuerzas productivas endógenas, como Karl Marx las llamaría (Moggach, 2013), es el significado concreto de perfección en este caso. Wolff justifica las políticas del despotismo ilustrado no por su contribución a la libertad de los sujetos, sino a su felicidad y perfección. Esta es precisamente la posición que Immanuel Kant denuncia por despótica en su texto importante de 1793, Teoría y práctica (Kant, 1923 [1793]: 290-291).

En respuesta a Leibniz, Kant rechaza las conclusiones perfeccionistas asumiendo ciertamente las implicaciones normativas del primer principio de la libertad espontánea, pero ofreciendo a la vez una nueva concepción de esta idea. Allí aparece un orden de derecho, entendido como ámbito de acciones externas espontáneas y mutuamente compatibles. Kant redefine el concepto de espontaneidad en una compleja crítica de la formulación leibniziana. La espontaneidad kantiana está vinculada a la libertad, no en el sentido de que exteriorice su contenido interior, como en Leibniz, sino en el sentido de que la voluntad no está ligada a ninguna causa ajena. Kant se refiere a esta capacidad como libertad negativa (Kant, 1908/1913 [1788]:33-35), la habilidad de la voluntad para abstraerse de causas externas, o bien admitirlas selectivamente como relevantes para la acción, de acuerdo a criterios racionales. Para Kant, estamos razonablemente influidos, pero no razonablemente determinados (Allison, 1990: 5-6, 39-40, 60-61, 191-98); mas tampoco precisamos de una ley monádica de desarrollo interior. He aquí nuestra espontaneidad, nuestra habilidad para marcarnos libremente las metas. La espontaneidad kantiana que se causa a sí misma debe guiarse por la ley moral y, en consecuencia, por la más elevada capacidad de ser autónomo y legislador de uno mismo, pero el ámbito del derecho, ahora distinto del de la moralidad, es el terreno en el cual la libertad de cada individuo para elegir sus fines se reconoce y armoniza en su aspecto exterior. Así emerge una tríada en la razón práctica: felicidad, derecho y virtud. Kant separa la búsqueda de perfección en dos componentes: por un lado, la felicidad material cae en la categoría de la razón práctica empírica y, por otro, la perfección moral-intelectual es considerada como razón pura práctica, figurando así en la esfera de la virtud, la cual no puede ser objeto de coacción. El ámbito del derecho (el cual está enraizado también en la razón pura práctica; pues debe haber derechos para que así se pueda reconocer la espontaneidad, aunque, en casos específicos, no necesitamos estar 
moralmente motivados para actuar correctamente) es el terreno sobre el que pueden desplegarse los principios que guían y limitan a los individuos en la selección de sus fines particulares o su propia felicidad, sin que exista una prescripción política de tales opciones. El primer principio leibniziano es restaurado en toda su vigencia; el segundo principio de justicia distributiva queda radicalmente subordinado a él; y el tercer principio, concebido como reino de los fines y auto-desarrollo individual y colectivo, se despolitiza de manera efectiva.

En este contexto, la relación oportuna entre libertad y justicia distributiva es acaloradamente discutida entre los kantianos: Humboldt defiende un Estado mínimo en su interpretación de Kant (Humboldt, 1851 [1792]); y Fichte contesta radicalmente a esta conclusión, también desde el espacio kantiano, aunque su propia solución sea problemática (Moggach, 2009). De acuerdo con los principios kantianos, el Estado no debe determinar legítimamente nuestros fines en lo relativo a la búsqueda de felicidad, cuya persecución no ha de impedir la libertad de los demás; aun así, el Estado, en cuanto orden de derecho, posee el deber de posibilitar a todos esta aspiración, para garantizar la justa distribución de los bienes de producción, de modo que cada uno pueda actuar de manera espontánea y ser capaz de vivir de su trabajo. Las intervenciones estatales conforme a este espíritu de reducir la pobreza, de eliminar el desempleo, etc., están diseñadas para promover el ejercicio del derecho, para dejar un espacio abierto a la acción espontánea, para liberar y no para fijar y satisfacer fines determinados. La justicia es condición universal del derecho o de la libertad.

Esta perspectiva arroja nueva luz sobre Hegel y su relación con la tradición postkantiana. Aunque el asunto es demasiado amplio como para ser abordado aquí, hay un elemento de particular interés, presente en la Filosofía del Derecho y en otros lugares, relativo a su polémica con la Escuela Histórica del Derecho, cuyos miembros dejan de lado el primer principio de Leibniz y ven el segundo como meramente descriptivo de las tradiciones históricas diferentes e igualmente válidas, sin superior justificación normativa. Hegel es un crítico muy duro del irracionalismo de este enfoque y sus implicaciones políticas en lo que tiene que ver con la justificación de las desigualdades sociales en el régimen pre-revolucionario (Hegel, 2009: \$258). Es más, la concepción hegeliana de la historia puede verse como la realización del tercer principio de Leibniz, entendido ahora como la historia de la libertad, y el despliegue del primer principio de la libertad espontánea. Sus discípulos de la izquierda continúan y elaboran este enfoque. 
En algunas propuestas, como en la de Bruno Bauer, la virtud debe ser repolitizada como precondición para la ciudadanía efectiva (Moggach, 2006). Bauer hará de la práctica rigurosa de la virtud algo central en su concepción del republicanismo moderno (Moggach, 2016). Él desarrolla un republicanismo perfeccionista postkantiano que impone un ideal rigorista de subordinación de lo particular a las demandas de lo universal; su teoría no identifica en primer lugar la libertad con la consecución de una hermosa armonía social, sino con la lucha heroica contra la dominación interior y exterior, contra el poder de los deseos no considerados por el sujeto, así como contra la hegemonía política de lo irracional o de lo tradicional. Que tal voluntad sublime y auto-transformación son imperativas para el progreso y la unidad social, constituye una conclusión del análisis de Bruno Bauer sobre el mundo de la Revolución Francesa y sus secuelas. Los intereses que conforman la sociedad civil moderna no son sólo diversos, sino que están encerrados en contradicciones recíprocas. Kant, y Schiller tras él, habían comprendido las interacciones en el ámbito jurídico como mutuamente limitantes, pero potencialmente reconciliables; la sociedad civil no parecía generar necesariamente intereses opuestos. En Schiller, los intereses se veían desde luego como fragmentarios, y además se afirmaban como unilaterales y en litigio, pero también eran potencialmente compatibles en su sustancia una vez que eran apropiadamente comprendidos y expuestos. La emergencia de la cuestión social, el reconocimiento de nuevas formas de pobreza urbana, desigualdad y opresión a comienzos del s. XIX, llevan a la conclusión contraria entre los miembros de la escuela hegeliana, y conducen a ulteriores reexámenes de las relaciones entre las esferas kantianas de la razón práctica. La invasión de la propiedad y el interés económico en el campo de la libertad política se convierte en una de las cuestiones teóricas fundamentales para la izquierda hegeliana, cuyas interpretaciones de Kant y Hegel pretenden impulsar la transformación social y la justicia frente al panorama del nuevo orden industrial emergente.

A diferencia de la conciliabilidad de los ámbitos externos de actividad postulados en la teoría jurídica de Kant, Bruno Bauer ve la sociedad civil profundamente marcada por intereses privados incompatibles (1842: 199 ff.; 1845). El problema político para él no consiste meramente en acomodar estos intereses mediante un compromiso y ajuste pragmáticos, o incluso a través del delicado equilibrio de una educación estética, como en Schiller. En su lugar, estos intereses deben modificarse antes de poder ser armonizados o, más bien, los portadores individuales de estos intereses deben cambiar ellos mismos, dado que el perfeccionismo postkantiano evita expresamente las 
intervenciones paternalistas. La determinabilidad de tales sujetos se aborda a través de categorías estéticas, en especial, la de lo sublime. En Bauer lo sublime aparece en dos dimensiones: subjetivamente, en el dominio de los intereses particulares del yo, lo cual conlleva un proceso de auto-transformación más severo que el concebido por Schiller, en tanto que el asunto no es ya la rigidez de los intereses, sino su inconmensurabilidad; y objetivamente, en la contribución a la lucha histórica en marcha por la realización de la libertad y la razón en la vida social y política (Moggach, 2003). Tal posición sitúa a Bauer mucho más próximo a la solución jacobina, a la cual Schiller había renunciado, en la medida en que la particularidad en sí misma puede presentarse como hostil a la libertad racional; pero Bauer sigue insistiendo en que los individuos deben emanciparse por sus propios medios de los intereses, deseos y filiaciones irracionales, ya que no pueden ser liberados por otro. No es el Estado el que libera a los sujetos, sino ellos los que se liberan en la lucha por la construcción del orden republicano.

La Modernidad para Bauer se presenta como una dura alternativa: es o una cultura de la división o de la libertad. Al romper el orden jerárquico tradicional de la sociedad, la Modernidad lanza a los individuos a la reconstrucción de las relaciones sociales, bien siguiendo simplemente el camino de los intereses privados en conflicto, o sometiendo a crítica estos intereses. Esta libertad crítica, denominada "autoconciencia universal”, estipula que los individuos no han de ser inmediatamente determinados por sus deseos, sino que deben ser capaces de emanciparse a sí mismos de sus intereses e identidades particulares allá donde estos entren en conflicto con el progreso del interés general. La crítica implica la evaluación teórica de los propios valores, así como la de las instituciones y prácticas sociales, además de un examen de su pretensión de validez; y también obliga a la intervención práctica, desafiando y expurgando todas las relaciones irracionales (Bauer, 1841; Moggach, 2003: 99-118). A través de su concepto de autoconciencia universal, Bauer adapta la razón práctica kantiana. Tomando el punto de vista del interés general, y discutiendo racionalmente sobre las máximas de su acción, los sujetos éticos muestran espontaneidad en la arena política, liberándose a sí mismos de la determinación irreflexiva de causas externas o pulsiones internas no consideradas. La virtud política es la sujeción de lo particular a la disciplina de lo universal. Los deseos, las pulsiones y los intereses privados no constituyen el más profundo y auténtico yo (Allison, 1990: 191-198), sino que son obstáculos para la libertad, en la medida en que implican una determinación heterónoma. 
Bauer extiende la idea de autonomía tomando las premisas morales de Kant como base para las acciones y relaciones jurídico-políticas. Así resitúa la virtud en el ámbito del derecho, de donde Kant la había sacado. La virtud política significa que los fines de la acción política (y también moral) exigen de legitimación universal y justificación racional, basada en la promoción de las condiciones para la libertad. El bienestar o la utilidad personal no deben anular las consideraciones sobre el bien general. Al reconocer la capacidad de todos los sujetos para reclamar la igualdad moral y jurídica, esta universalidad repudia las distinciones de rango, estatus, derechos y privilegios heredados que se asocian al viejo orden pre-revolucionario (Bauer, 1843). En la visión de Bauer, estos deben ser eliminados como meros vestigios históricos, sin ninguna justificación racional.

El interés económico de los individuos emancipados modernos también debe someterse a crítica; el desplazamiento de la virtud hacia el derecho se debe a una nueva concepción de la sociedad civil y sus límites, y no se trata de una regresión teórica a tiempos anteriores a Kant. La concepción de la izquierda hegeliana sobre la oposición entre ciudadanía e individualismo egoísta se fragua al considerar los problemas y características de la sociedad civil moderna. La amenaza para la libertad proviene ahora de la tendencia de la propiedad a desfigurar el dominio de lo político. Los individuos quedan inmovilizados en su esfera de interés privado, irresolublemente enfrentados entre ellos y opuestos al progreso histórico, al tiempo que el Estado autoritario se apropia de lo universal como un poder trascendente, actuando al servicio de los grupos dominantes. En este enfoque, la virtud y el comercio están en conflicto porque el mercado promueve la heteronomía y la oposición de intereses. Esto inclina a los sujetos a maximizar la propiedad en detrimento de sus compromisos políticos. Se trata de una repetición de la antigua crítica republicana a la crematística (Hont e Ignatieff, 1983; Ottow, 1996), que Bauer intenta reivindicar a través de sus reflexiones sobre el surgimiento del Estado y la economía modernas. Originada como una lucha de emancipación contra los privilegios y la jerarquía irracional, la Revolución Francesa se convirtió, después del derrocamiento de los jacobinos, en un vehículo para la rapiña y la conquista imperial al servicio de la burguesía francesa (Bauer et al., 1843-1844) . El mundo post-revolucionario, según Bauer, está al borde de la disolución en una informe sociedad de masas. Los individuos en un mundo como este son particularistas, pero inconsistentes, en la persecución de sus intereses inmediatos: renuncian al potencial de espontaneidad y autonomía que la Modernidad posibilita de forma excepcional. La virtud republicana 
debe vencer a estas nuevas formas de heteronomía, haciendo realidad la autonomía en la práctica del derecho y la búsqueda de felicidad. Bauer pone en contraste a una ciudadanía virtuosa, o al pueblo como entidad política auto-determinada, frente a la sociedad de masas, partiendo del punto de vista de que la primera se ha inmunizado a sí misma contra el efecto disolvente y excluyente de la propiedad y el interés privado, con el propósito de actuar decisivamente y con determinación en pos del interés general. El futuro Estado republicano debe asegurar la extensión de las relaciones de derecho, reciprocidad y justicia en todos los ámbitos de actividad. El ejercicio del derecho está para reformar las instituciones del bienestar.

El programa republicano de Bauer en el fermento revolucionario de la década de 1840 fue una viva defensa de la soberanía popular. Era un firme rechazo al compromiso con el Estado monárquico en su engañosa envoltura reformista. Fue una crítica al liberalismo en cuanto mera traslación política del interés privado, y porque estaba además íntimamente comprometido con el viejo orden en razón de sus preocupaciones por la propiedad. Y finalmente fue un polémico combate con el socialismo emergente por ser incompatible con la libertad individual y la autodeterminación (Bauer, 1972: 526-529). Todas estas posiciones comparten una base común: el rechazo del particularismo y la defensa del interés universal contenido en la docrtrina de la autoconciencia infinita. Para Bauer la fuerza del particularismo sólo puede ser derrotada por la sublime determinabilidad del yo, un proceso de severa auto-transformación. Un rigorismo republicano es necesario en unas condiciones en las que los intereses privados se oponen diametralmente.

Los escritos de Bauer posteriores a las fallidas revoluciones de 1848 son altamente problemáticos. Sin embargo, su crítica de los años cuarenta es de un interés duradero. Identifica formas de dominación y heteronomía ocultas en las relaciones económicas contemporáneas, y defiende opciones republicanas modernas: la promoción y extensión del ámbito del derecho, y las virtudes de la ciudadanía activa. El republicanismo de Bauer no es alegremente optimista; invita a los sujetos a adoptar un ideal sublime y altamente exigente de auto-transformación, sin ofrecer garantías metafísicas de éxito. Se trata más bien de sacar nuevas prácticas y comprensiones de la libertad, bajo la idea guía de que "nada es imposible para el espíritu" (Bauer, 1968: 195).

Un segundo modelo de perfeccionismo postkantiano es el que caracteriza a las primeras obras de Karl Marx. Marx comparte con Bruno Bauer y la tradición idealista leibniziana el énfasis en la auto-actividad, que subyace en su idea del trabajo (Stedman Jones, 2016: 220). Esto es fundamental en su crítica 
del capitalismo como un sistema que no sólo depaupera a los trabajadores, privándoles de satifacciones materiales, sino que, en primer lugar, vicia su libertad, su capacidad para autodeterminarse en el trabajo. Marx contrasta su emergente materialismo militante con el sensualismo de Ludwig Feuerbach, argumentando que el defecto de este último es su fracaso a la hora de reconocer el poder emancipatorio del trabajo. Feuerbach no celebra la transformación sino el asentamiento, la pasividad, la receptividad de los sentidos: la inmersión de los seres humanos en la naturaleza y su dependencia de ella, más que su capacidad para transformarla mediante el trabajo. El propio Feuerbach cree que este desplazamiento hacia el naturalismo representa un avance teórico con respecto a las mistificaciones idealistas, en las cuales, según él, el pensamiento se separa de su raíz en los sentidos y el mundo natural (Feuerbach, 1981a; 1981b; 1981c) pero, como apunta Marx críticamente, la unidad del sujeto humano con la naturaleza se consigue a un alto coste, perdiéndose el sentido dinámico de la subjetividad, la auto-actividad y el hacerse a uno mismo, es decir, el elemento clave de la herencia idealista. Marx quiere mantener este sentido, pero de forma materializada en una teoría del trabajo (Marx, 1976b: 3-5).

La comprensión marxista del trabajo incorpora elementos de la tradición del idealismo alemán, sobre todo, las ideas de espontaneidad y auto-formación, que se derivan de la crítica de Leibniz al materialismo mecanicista (Bodéüs, 1993; Antognazza, 2009: 52-4). Al igual que en la idea de espontaneidad de Leibniz como acción que se auto-origina y no es una mera reacción a causas externas, cierta noción de causalidad inmanente subyace en la visión del trabajo no-alienado de Marx. Estas ideas se desarrollan más allá bajo la influencia de la razón práctica kantiana, con el acento sobre la autonomía o la autodeterminación racional, y su repudio de la heteronomía (Kant, 1903/1911 [1785]: 444-445); es desde este punto de vista como mejor se revela la especificidad de la teoría de la alienación de Marx. Siguiendo a Fichte, Marx vincula la espontaneidad y la autonomía con el trabajo, y examina las condiciones concretas para la actividad autodeterminada.

Para Fichte el trabajo es la capacidad de traducir nuestros fines y conceptos al mundo sensible, sometiendo el dominio de la objetividad a la luz de la razón práctica; el derecho al trabajo es el derecho natural fundamental (Fichte, 1971b: 9). En una sociedad justa, todos deben ser capaces de vivir, y vivir decentemente de su trabajo; así como poseer suficientes recursos e instrumentos como para sostener su actividad. Fichte detalla un Estado altamente intervencionista que, a pesar de sus enormes controles y restricciones, 
intenta promover la libertad de sus miembros y mantener las condiciones de su actividad, la capacidad para dar forma a la objetividad a la luz de sus ideas. Este Estado no está principalmente diseñado para vehicular la felicidad, como lo había estado el antiguo intervencionismo de Christian Wolff (Fichte, 1971a; Moggach, 2009). En tanto que deriva en primer lugar de consideraciones de la razón pura práctica y no de la razón empírica, y persigue las condiciones para su ejercicio efectivo, el Estado fichteano es representativo del perfeccionismo postkantiano. Marx adapta estos temas a su propia crítica político-económica. Además, siguiendo a Hegel y la recepción izquierdista del Vormärz, Marx busca desvelar las dinámicas y contradicciones internas que definen el mundo moderno, con sus características nociones de sociedad civil y Estado, y dar cuenta de sus negaciones determinadas, de sus condiciones necesarias de transformación.

Desde el otoño de 1843, Marx toma la auto-emancipación de la clase trabajadora como la característica esencial de su versión específica del socialismo. Al reflexionar sobre las relaciones concretas de la sociedad civil, Marx da forma a su teoría del trabajo en los Manuscritos de París de 1844 (Marx, 1975a) basándose en las consideraciones de Hegel en torno a la teleología exterior en la Ciencia de la Lógica (Hegel, 1969: 735-754). Como acción teleológica, el proceso de trabajo abarca tres momentos: en primer lugar, la finalidad subjetiva, o la intención que precede, anima y dirige las etapas de su propia realización; después, los medios o la instrumentalidad mediante la cual la finalidad subjetiva se lleva a cabo, la aplicación de herramientas y mecanismos causales para transformar la objetividad a la luz del fin propuesto; y finalmente, el objetivo o fin realizado, el producto de la actividad, en el cual la idea que inicia el proceso se hace ahora concreta. Al darse en cada uno de estos tres aspectos, la alienación del trabajo es la subversión de la conexión entre el sujeto activo y los propósitos que persigue en su actividad; esta es la reconfiguracción materialista que hace Marx de la distinción kantiana entre autonomía y heteronomía, entre los actos auto-dirigidos o los dirigidos por otros. Prescrita por una subjetividad ajena, la finalidad del trabajo bajo las actuales relaciones de producción es heterónoma, y viola el principio de auto-actividad que Marx considera la esencia de la libertad. El trabajo está alienado porque los proletarios como sujetos activos no pueden determinar sus propias intenciones en el trabajo; ejecutan una finalidad heterónoma, impuesta por los propietarios del aparato productivo. Están, además, privados de acceso a los intrumentos de trabajo, que son propiedad privada de otra clase. Esta relación de exclusión con respecto a los medios de producción 
define la misma existencia del proletariado como clase social. Tal y como Marx argumentará en La ideología alemana, esto marca la especificidad histórica de esta clase respecto a otros grupos dominados, como los esclavos de la Antigüedad (totalmente reducidos a un estatus instrumental: organa empsycha, como los llama Aristóteles) y los siervos del feudalismo (pequeños productores independientes, pero mantenidos en situación de sometimiento por los propietarios de la tierra) (Marx y Engels, 1975: 34-35). Los proletarios encarnan la heteronomía al convertirse en instrumentos de la voluntad de otro durante el proceso de trabajo (aunque, a diferencia de los esclavos, los proletarios son dueños de sí mismos como precondición para intercambiar su fuerza de trabajo por un salario; su emancipación jurídica, aunque históricamente supone un avance, es la condición de su verdadera explotación económica) (Marx, 1967a: 119-125). Están, en tercer lugar, privados del producto de su trabajo, que pertenece al dueño de los medios de producción. En el trabajo, el proletariado renueva constantemente las propias condiciones que le subyugan; reproduce el gigantesco sistema de heteronomía como producto de su propia acción (Quante, 2009; Marcuse, 1973; Arthur, 1986). Aun así, en tal centralidad descansa el potencial transformador del proletariado. El rasgo esencial del capitalismo es la separación de los trabajadores de los medios de producción. Esta característica es la que define al sistema y también marca su límite, las condiciones y el factor de su posible derrocamiento.

El socialismo, o la autonomía del trabajo, significa la superación de la alienación en cada uno de los tres momentos que Marx identifica en los Manuscritos de París. Implica que los trabajadores adquieren el control sobre las etapas del intercambio material que llevan a cabo con la naturaleza: la determinación de los objetivos a alcanzar en el trabajo, los procesos e instrumentos implicados en la realización de esos fines, y la distribución de los productos. Esto exige, según Marx, la propiedad colectiva de los medios de producción. La imposición de los objetivos del trabajo por los actuales propietarios del aparato productivo impide la autodeterminación del sujeto en el trabajo, viola su libertad, y no es simplemente una humillación para su felicidad o satisfacción material. De otra manera, para Marx un mero socialismo distributivo, que persiguiera aumentar los salarios o el nivel de consumo de los trabajadores, no sería suficiente (Marx, 1976a; Stedman Jones, 2016: 642). Tal solución, aunque sea importante, es incompleta para la visión de Marx, pues dejaría intactas las relaciones de dominación en el mismo acto productivo. No es simplemente cuestión de mejorar las condiciones de vida y trabajo del proletariado, sino de transformar su relación con el trabajo mismo en 
todas sus fases. Aunque evita el lenguaje idealista, Marx está implícitamente apuntando a la distinción kantiana entre el domino de la libertad, en el que los sujetos colocan sus propias metas, y el de la felicidad, o el grado de satisfacción de las necesidades. Esto último no es baladí para el socialismo marxista (y mucho menos para Kant), pero tampoco es primordial; o mejor dicho, como Marx formulará más tarde mediante su conocida distinción, las relaciones de producción (el momento de la actividad formativa) condicionan y son la base de las relaciones de distribución (el reparto del producto) (Marx, 1973: 81-114). El fracaso a la hora de reconocer esta distinción es la raíz del socialismo sentimental de la década de 1840, y más tarde, de eso que Marx siempre desprecia: el socialismo que se centra en la desgracia y miseria de los trabajadores, más que en su reducción a instrumentos dependientes de la voluntad de otro y en su potencial revolucionario para reclamar su propia actividad. Al criticar a los socialistas que describen al proletariado como una víctima necesitada y sufriente, Marx, aunque nunca minimiza la pobreza de los trabajadores, pone sobre todo el acento en el rol histórico de esta clase como dinamizadora de la transformación del mundo (Marx y Engels, 2002: 245-257). Es un poder activo, formativo, la negación determinada de las relaciones de producción existentes (Marx, 1975b: 175 ff.). Al recoger el legado del idealismo, en el cual la razón práctica empírica y la felicidad están gobernadas por la idea de libertad prescrita por la razón pura práctica, Marx reconoce la dualidad del trabajo como satisfacción de una necesidad y como actividad auto-causada o auto-dirigida. Evita así los defectos de un materialismo como el de Feuerbach, esto es, la negación de la espontaneidad subjetiva. Para Marx, los idealistas habían fracasado al pensar el principio de autonomía hasta sus últimas consecuencias poniendo el énfasis solo, por más que en parte también correctamente, en la primacía de la actividad. Esta actividad necesita ahora ser reinterpretada como trabajo, como intercambio material con la naturaleza. Es aquí, más que en la ética o en la política, donde la verdadera autonomía se conquista, en el control colectivo de los medios de producción.

A pesar de su rechazo al moralismo como una forma insípida e impotente de crítica, el programa de emancipación de Marx no está vacío de contenido ético. Su posición en la época del Vormärz ha sido descrita como una teoría de la libertad en cuanto auto-realización (Brudney, 1998: 160-167), o como una reflexión sobre las necesidades dinámicas (Leopold, 2007: 226), que incluye tanto componentes naturales como históricamente variables (necesidades físicas básicas, desarrollo intelectual, trabajo y creatividad e inclusión en 
la comunidad) (ib.: 224). Su esencia es el reconocimiento de la auto-actividad y de las condiciones de su efectividad, la idea central de los perfeccionismos postkantianos de Fichte y de la izquierda hegeliana (Moggach, 2011; Quante, 2006). La postura de Marx en el Vormärz tiene características distintivas, sin embargo. Esto condiciona la subsecuente evolución de su pensamiento después de 1848.

El primer pensamiento de Marx combina las ideas kantianas sobre la libertad con la idea de un bien sustantivo que recuerda a perfeccionismos anteriores (McCarthy, 1992; Stedman Jones, 2002). Lo que él retiene de Kant y Fichte es la idea de la espontaneidad de los agentes, la de su capacidad (a través del trabajo) para iniciar cambios en el mundo objetivo y llevar a cabo sus fines subjetivos. En su concepción de la época del Vormärz, el trabajo es el vehículo tanto para la satisfacción de las necesidades como para la libertad. En sus obras posteriores, el propio Marx pasa por alto esta dualidad fundamental cuando asimila el trabajo al reino de la necesidad, al ámbito de la razón práctica empírica y sus exigencias. Entonces evoca el reino de la libertad más allá del trabajo, como una posibilidad sólo alcanzable una vez que el ser humano se ha emancipado del trabajo, pero no contenida en el trabajo mismo (Marx, 1967b: 820; Sayers, 2006); mas aquí él renuncia también a su visión anterior de la alienación como fundamentalmente un asalto a la libertad de los productores en el acto de trabajo.

Pero ya en sus escritos más tempranos, el giro de Marx hacia el materialismo es en algunos aspectos una regresión a formas de perfeccionismo anteriores a Kant. Estos elemenos prekantianos llegan a Marx a través de la idea feuerbachiana de Gattungswesen (ser genérico) y las condiciones que postula para el correcto desarrollo, las cuales, a pesar de la crítica marxista a la pasividad feuerbachiana, aún se mantienen en su noción más dinámica de autoformación (Quante, 2009: 264-268). Al hacer énfasis en los valores colectivos compartidos, en la efectividad de los fines dados o predeterminados (incluso si estos se conciben como históricamente variables más que biológicamente fijos), Marx fracasa al recoger aspectos significativos de la visión kantiana sobre la actividad, en especial, la función evaluadora y selectiva de la libertad negativa, que otros miembros de la izquierda hegeliana sí pusieron de relieve. Él obvia el momento vital, "formal”, de la emancipación que Bauer reconoce, sobre todo, en la centralidad del asentimiento individual a los fines, es decir, la manera en que estos son postulados, interiorizados y convertidos en guías de acción (Kant, 1903/1911 [1785]: 444). La consecuencia es que, para Marx, lo universal permanece como una propiedad del género, y el concepto 
dialéctico de singularidad como síntesis de lo universal y lo particular está mal definido. Esta deficiencia es en parte responsable de la degradación de los derechos y la ciudadanía en el pensamiento de Marx; los individuos son parte de un todo, más que agentes auto-definidos, pese al efecto compensatorio de la visión del trabajo de Marx. La cualidad genérica o distributiva de lo universal también está tras la idea de que el Estado es prescindible bajo el socialismo, en tanto que no es necesario ningún foro especial para el ejercicio de la libertad una vez que la esfera de la producción está sometida al control social. Las primeras críticas de Marx al republicanismo ponen de manifiesto estas conclusiones (Moggach, 2018).

El nexo entre el trabajo y la libertad es la conexión fundamental entre Marx y el idealismo alemán. Según la idea que él tiene de sí mismo, Marx rompe con esta tradición. Aun así, su materialismo, al poner el foco en la actividad espontánea y en el poder formativo de la acción humana a la hora de moldear la objetividad, posee una estrecha afinidad con la concepción idealista de la acción, incluso cuando Marx rechaza el enclaustramiento del idealismo en el reino del pensamiento. El determinismo de Marx es igualmente ambiguo: en un sentido amplio, el ser determina al pensamiento (quizás interpretable desde una perspectiva kantiana como la indicación de que la intuición precede y determina al concepto) y, en un sentido más específico, está la idea del determinismo económico (de las instituciones y las acciones), cuyo rigor y rango de aplicación pemanece incierto. Si lo que aquí está en juego es la negación o problematización de la libertad negativa kantiana por parte de Marx, esto es, de la capacidad de los individuos para abstraerse de causas externas $\mathrm{y}$ admitirlas selectivamente en sus acciones de acuerdo a un criterio racional, puede concluirse que Marx está enfrentando un sentido de la espontaneidad contra otro, a saber, contra la capacidad para exteriorizar nuestras metas en el mundo objetivo, un postulado fundamental del idealismo, que continúa siendo esencial en sus consideraciones sobre el trabajo y la libertad.

El legado del idealismo, así como de los programas éticos postkantianos, es la ampliación activa, formativa, espontánea para el sujeto, del reino de la libertad. Esta es la idea distintiva de los perfeccionismos postkantianos, y si bien Marx la enriquece mediante su análisis del trabajo, también fracasa al sacar todas sus implicaciones para una vida social emancipada. La visión de Marx del proceso histórico y su crítica del capitalismo ofrecen poderosas revelaciones sobre la negatividad implícita en el mundo moderno, también sobre la heteronomía y autonomía del trabajo; pero estas aportaciones, esta grandeza, se deriva de lo que Marx retiene del idealism, y sus defectos pro- 
vienen de aquello en lo que él se desvía de este. Los dos modelos de perfeccionismo postkantiano esbozados aquí son ilustrativos de la amplitud de este pensamiento y de la fuerza de su aplicación a cuestiones sociales concretas.

\section{REFERENCIAS BIBLIOGRÁFICAS}

Allison, H. E. (1990). Kant's Theory of Freedom. Cambridge: Cambridge University Press.

AntognazZa, M. R. (2009). Leibniz. An Intellectual Biography. Cambridge: Cambridge University Press.

ArThur, C. J. (1986). Dialectics of Labour: Marx in bis Relation to Hegel. London: Blackwell.

Bauer, B. (anon.) (1841). Die Posaune des jüngsten Gerichts über Hegel den Atheisten und Antichristen: Ein Ultimatum. Leipzig: Otto Wigand.

BAuer, B. (anon.) (1842). Die gute Sache der Freibeit und meine eigene Angelegenheit. Zurich and Winterthur: Verlag des literarischen Comptoirs.

Bauer, B. (anon.) (1843). Geschichte der Politik, Kultur und Aufklärung des achtzebnten Jabrbunderts, Erster Band: Deutschland wäbrend der ersten vierzig Jabre des acbtzehnten Jahrbunderts. Charlottenburg: Verlag von Egbert Bauer.

BAuer, B. (anon.) (anon.). (1845). "Das Wohl der arbeitenden Klassen”. Norddeutsche Blätter 9, 52-66.

BAUER, B. (anon.) (1968). "Die Fähigkeit der heutigen Juden und Christen, frei zu werden”. En Feldzüge der reinen Kritik. Ed. H.-M. Sass. Frankfurt am Main: Suhrkamp.

BAuer, B. (anon.) (1972). “Erste Wahlrede von 1848”. En Barnikol, E. Bruno Bauer: Studien und Materialien. Ed. P. Riemer y H.-M. Sass. Assen: Van Gorcum, 526529.

BAUER, B. et al (1843-4). Denkwürdigkeiten zur Geschichte der neweren Zeit seit der Französischen Revolution. Nach den Quellen und Original Memoiren bearbeitet und herausgegeben von Bruno Baner und Edgar Bawer. Charlottenburg: Verlag von Egbert Bauer.

Bodéüs, R. (1993). Leibniz-Thomasius. Correspondances. Paris: Vrin.

Brudney, D. (1998). Marx's Attempt to Leave Philosophy. Cambridge, MA: Harvard University Press.

CASSIRER, E. (1962 [1902]). Leibniz' System in seinen wissenschaftlichen Grundlagen. Hildesheim: Olms.

CAssirer, E. (2001 [1917]). Freibeit und Form. Studien zur deutschen Geistesgeschichte. Hamburg: Meiner.

Feuerbach, L. (1981a). "Grundsätze der Philosophie der Zukunft”. En Schuffenhauer, W. (ed.). Gesammelte Werke, Vol. 9. Berlin: Akademie Verlag, 264-341. 


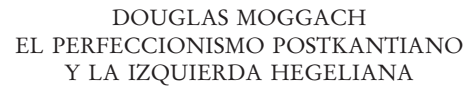

Feuerbach, L. (1981b). "Vorläufige Thesen zur Reformation der Philosophie”. En Schuffenhauer, W. (ed.). Gesammelte Werke, Vol. 9. Berlin: Akademie Verlag, 243-263.

Feuerbach, L. (1981c). "Zur Kritik der Hegelschen Philosophie”. En Schuffenhauer, W. (ed.). Gesammelte Werke, Vol. 9. Berlin: Akademie Verlag, 16-62.

Fichte, J. G. (1971a). Der geschloßne Handelsstaat. En Werke, Bd. III. Berlin: de Gruyter.

Fichte, J. G. (1971b). System der Sittenlehre. En Werke, Bd. IV. Berlin: de Gruyter. GuYer, P. (2011). "Kantian Perfectionism”. En Jost, L.; Wuerth, J. (eds.). Perfecting Virtue. New Essays on Kantian Ethics and Virtue Ethics. Cambridge: Cambridge University Press, 194-214.

Hegel, G. W. F. (1969). Science of Logic. London: Allen and Unwin.

Hegel, G. W. F. 2009). Grundlinien der Philosophie des Rechts. En Gesammelte Werke. Hamburg: Meiner.

HonT, I.; Ignatieff, M. (eds.). (1983). Wealth and Virtue: The Shaping of Political Economy in the Scottish Enlightenment. Cambridge: Cambridge University Press.

KANT, I. (1903/1911 [1785]). Grundlegung zur Metaphysik der Sitten. En Immanuel Kants Gesammelte Schriften, Vol. 4. Berlin: de Gruyter.

KanT, I. (1908/1913 [1788]). Kritik der praktischen Vernunft. En Immanuel Kants Gesammelte Schriften, Vol. 5. Berlin: de Gruyter.

Kant, I. (1923 [1793]). "Über den Gemeinspruch: 'Das mag in der Theorie richtig sein, taugt aber nicht für die Praxis'”. En Immanuel Kants Gesammelte Schriften, Vol. 8. Berlin: de Gruyter.

KLIPPEL, D. (1998). “Der liberale Interventionsstaat. Staatszweck und Staatstätigkeit in der deutschen politischen Theorie des 18. und der ersten Hälfte des 19. Jahrhunderts". En Lück, H. (ed.). Recht und Rechtswissenschaft im mitteldeutschen Raum. Köln: Böhlau.

Leibniz, G. W. (1990 [1667]). Nova Methodus Discendae Docendaeque Jurisprudentiae. En Sämtliche Schriften und Briefe. Berlin: Akademie Verlag.

Leibniz, G. W. (1991 [1720]). Monadology. Ed. Nicholas Rescher. Pittsburgh: University of Pittsburgh Press.

Leopold, D. (2007). The Young Karl Marx. German Philosophy, Modern Politics, and Human Flourishing. Cambridge: Cambridge University Press.

Maesschalck, M. (1996). Droit et création sociale chez Fichte: une philosophie moderne de l'action politique. Louvain: Peeters.

Marcuse, H. (1973). "The Foundations of Historical Materialism”. En Studies in Critical Philosophy. Boston: Beacon Press.

Marx, K. (1967a). Capital, Vol. 1. New York: International Publishers.

MarX, K. (1967b). Capital, Vol. 3. New York: International Publishers.

Marx, K. (1973). Grundrisse. London: Penguin. 
Marx, K. (1975a). “Economic and Philosophical Manuscripts of 1844”. En Collected Works, Vol. 3. New York: International Publishers, 270-282.

Marx, K. (1975b). "Hegel's Philosophy of Law. Introduction”. En Collected Works, Vol. 3. New York: International Publishers, $175 \mathrm{ff.}$

MarX, K. (1976a). “The Historiography of True Socialism”. En Collected Works, Vol. 5. New York: International Publishers, 516-519.

Marx, K. (1976b). “Theses on Feuerbach”. En Collected Works, Vol. 5. New York: International Publishers, 3-5.

Marx, K.; Engels, F. (1975). The German Ideology. En Collected Works, Vol. 5. New York: International Publishers.

Marx, K.; Engels, F. (2002). The Communist Manifesto. Ed. Gareth Stedman Jones. Harmondsworth: Penguin.

McCarthy, G. E. (1992). Marx and Aristotle. Lanham, MD: Rowman and Littlefield.

McCarthy, G. E. (2015). "The Last of the Schoolmen. Natural law and Social Justice in Karl Marx”. En Thompson, M. J. (ed.), Constructing Marxist Ethics. Critique, Normativity, Praxis. Leiden: Brill.

Moggach, D. (2003). The Philosophy and Politics of Bruno Baner. Cambridge: Cambridge University Press.

Moggach, D. (2006). "Republican Rigorism and Emancipation in Vormärz Germany”. En Moggach, D. (ed.). The New Hegelians. Politics and Philosophy in the Hegelian School. Cambridge: Cambridge University Press, 114-135.

Moggach, D. (2009). "Freedom and Perfection: German Debates on the State in the Eighteenth Century". Canadian Journal of Political Science, Vol. 42, no. 4, 1003-1023.

Moggach, D. (2011). “Post-Kantian Perfectionism”. En Moggach, D. (ed.). Politics, Religion, and Art. Hegelian Debates. Evanston IL: Northwestern University Press, 179-200.

Moggach, D. (2013). “German Idealism and Marx”. En Boyle, N.; Walker, J. (eds.). The Impact of Idealism - the Legacy of Post-Kantian German Thought, Vol. 2, Historical, Social, and Political Theory. Cambridge: Cambridge University Press, 82-107.

Moggach, D. (2016). “Die Kultur der Zerrissenheit und ihre Aufhebung”. En Schmidt am Busch, H.C. (ed.). Die Philosophie des Marktes, Deutsches Jabrbuch Philosophie, Vol. 7, 71-98.

Moggach, D. (2018). "German Republicans and Socialists in the Prelude to 1848”. En Moggach, D.; Stedman Jones, G. (eds.). The 1848 Revolutions and European Political Thought. Cambridge: Cambridge University Press, 216-235.

Oттош, R. (1996). Markt, Tugend, Republik. Berlin: Akademie Verlag.

Quante, M. (2006). “Die fragile Einheit des Marxschen Denkens”. Zeitschrift für philosophische Forschung 60, 591-608. 
Quante, M. (2009). “Kommentar”. En Marx, K. Ökonomisch-philosophische Manuskripte. Frankfurt/M: Suhrkamp, 247-262.

Rutherford, D. (2005). "Leibniz on Spontaneity”. En Rutherford, D.; Cover, J.A. (eds.). Leibniz. Nature and Freedom. Oxford: Oxford University Press, 156-180.

SAYERS, S. (2006). "Freedom and the 'Realm of Necessity". En Moggach, D. (ed.). The New Hegelians. Politics and Philosophy in the Hegelian School. Cambridge: Cambridge University Press, 261-274.

Schneewind, J.B. (1998). The Invention of Autonomy. Cambridge: Cambridge University Press.

SCHNEIDER, H.-P. (1967). Justitia universalis. Quellenstudien zur Geschichte des "christlichen Naturrechts" bei Gottfried Wilhelm Leibniz. Franfurt am Main: Klostermann.

Stedman Jones, G. (2016). Karl Marx. Greatness and Illusion. Cambridge MA: Harvard University Press.

- (2002). "Introduction". En Marx, K.; Engels, F. The Communist Manifesto. Harmondsworth: Penguin, 99-140.

Stipperger, E. (1984). Freiheit und Institution bei Christian Wolff (1679-1754). Frankfurt: Lang.

Tribe, K. (1988). Governing Economy. The Reformation of German Economic Discourse, 1750-1840. Cambridge: Cambridge University Press.

Von Humboldt, W. (1851 [1792]). Ideen zu einem Versuch, die Gränzen der Wirksamkeit des Staats zu bestimmen. Breslau: Trewendt.

WolfF, C. (1969 [1754]). Institutiones juris naturae et gentium. Gesammelte Werke. Ed. M. Thomann. Hildesheim: Olms. 
